\title{
Quasinormal modes and stability of the rotating acoustic black hole: numerical analysis
}

\author{
Vitor Cardosd* \\ Centro de Física Computacional, Universidade de Coimbra, P-3004-516 Coimbra, Portugal \\ José P. S. Lemođ \\ Centro Multidisciplinar de Astrofísica - CENTRA, \\ Departamento de Física, Instituto Superior Técnico, \\ Universidade Técnica de Lisboa, Av. Rovisco Pais 1, 1049-001 Lisboa, Portugal \\ Shijun Yoshidd \\ Science and Engineering, Waseda University, Okubo, Shinjuku, Tokyo 169-8555, Japan
}

(Dated: August 24, 2018)

\begin{abstract}
The study of the quasinormal modes (QNMs) of the $2+1$ dimensional rotating draining bathtub acoustic black hole, the closest analogue found so far to the Kerr black hole, is performed. Both the real and imaginary parts of the quasinormal (QN) frequencies as a function of the rotation parameter $B$ are found through a full non-linear numerical analysis. Since there is no change in sign in the imaginary part of the frequency as $B$ is increased we conclude that the $2+1$ dimensional rotating draining bathtub acoustic black hole is stable against small perturbations.

PACS numbers: 04.70.-s
\end{abstract}

\section{INTRODUCTION}

A guitar string in vacuum vibrates in normal modes. However, a guitar string immersed in air vibrates truly in quasinormal modes (QNMs) since it looses energy to sound waves. The same happens to an acoustic black hole. Due to energy losses to the medium in sound wave acoustic perturbations, its modes of vibration are quasinormal (QN). This QN behavior is expected, as acoustic black holes are real analogues of true general relativistic black holes which are known to vibrate in a QN form due to losses in gravitational and other types of radiation to infinity.

Acoustic black holes and their analogies with true black holes were found by Unruh by noticing that the equations of motion for sound waves in a background fluid flow and the equations of motions for a scalar field in a black hole background are similar [1]. The background fluid flow acts as an effective black hole metric implying the existence of a sound horizon, the surface where the velocity of the flow is equal to the velocity of sound in the medium. A number of phenomena that occurs with true black holes also occurs with acoustic black holes. For instance, Hawking radiation is now phonon radiation [2, 3, 4, 5]. Geodesic and causal structure can also be studied as was done in [6] where Penrose-Carter diagrams for several effective acoustic spacetimes were drawn. Black holes in other analogue systems, like condensed matter ones, can also be studied (see [7, 8, [9] to

\footnotetext{
*Electronic address: vcardoso@teor.fis.uc.pt

${ }^{\dagger}$ Electronic address: lemos@fisica.ist.utl.pt

${ }^{\ddagger}$ Electronic address: shijun@waseda.jp
}

name a few).

In the study of true general relativistic black holes QNMs are important for a number of reasons. The most important is that they provide means to identifying black hole parameters like the mass and angular momentum, since the real and imaginary part of the $\mathrm{QN}$ frequencies, which give the vibration frequency and the exponential damping frequency respectively, depend only on those parameters. Through their study one further tests the stability of the system, as any imaginary frequency with the wrong sign would mean an exponentially growing mode, rather than damping. QNMs have also been connected to the quantization of the black hole area, where it seems that the highly damped modes, those that are almost instantaneous, are associated with transitions between area levels at large quantum numbers (see 10 for a review on QNMs and a list of complete citations therein). Since QNMs appear naturally in general relativistic black holes, they also should appear in their analogues, the acoustic black holes.

The study of QNMs of the $2+1$ dimensional rotating draining bathtub acoustic black hole [4, 12], the closest analogue found so far to the Kerr black hole (see 11 for developments on this subject), was initiated in 13. through the use of a WKB approximation, valid for small black hole rotation. Various interesting points besides QNM behavior, like late-time tails and superradiance, were also discussed. In relation to large damped QNMs it was found that there are no asymptotic QN frequencies, a puzzling result if connected to the area quantization issue. Moreover, the WKB scheme used in 13] gave indications that, due to a possible change in the sign of the imaginary part of the frequency $\omega$, this $2+1$ acoustic black hole could become unstable at large rotation parameter $B$, or at large azimuthal number $m$. Recent 
work on this [14], making use of a matching procedure, is also not appropriate to give a convincing stability proof. Is the $\operatorname{Im} \omega$ part lower, equal or greater than zero for $B$ or $m$ large? In this paper we make a full numerical analysis to settle this issue. We show that the instability never sets in.

\section{FORMALISM}

The draining bathtub model is a $2+1$ dimensional flow with a singular vortex-like sink (or source) at the origin. The acoustic metric describing the propagation of sound waves in this fluid flow is $[4$ :

$$
\begin{aligned}
d s^{2}= & -\left(c^{2}-\frac{A^{2}+B^{2}}{r^{2}}\right) d t^{2} \\
& +\frac{2 A}{r} d r d t-2 B d \phi d t+d r^{2}+r^{2} d \phi^{2} .
\end{aligned}
$$

Here $A$ and $B$ are arbitrary real positive constants related to the radial and angular components of the background fluid velocity:

$$
\vec{v}=\frac{-A \vec{r}+B \vec{\phi}}{r},
$$

where $\vec{r}$ and $\vec{\phi}$ are orthogonal unit basis vectors pointing along the axes. It is however better to work with a more transparent metric. Some physical properties of our draining bathtub metric are more apparent if we cast the metric in a Kerr-like form performing the following coordinate transformation (see 12, 13]):

$$
\begin{aligned}
d t & \rightarrow d \tilde{t}=d t-\frac{A r}{r^{2} c^{2}-A^{2}} d r \\
d \phi & \rightarrow d \tilde{\phi}=d \phi-\frac{B A}{r\left(r^{2} c^{2}-A^{2}\right)} d r,
\end{aligned}
$$

Then the effective metric takes the form

$$
\begin{aligned}
d s^{2}= & -\left(1-\frac{A^{2}+B^{2}}{c^{2} r^{2}}\right) c^{2} d \tilde{t}^{2} \\
& +\left(1-\frac{A^{2}}{c^{2} r^{2}}\right)^{-1} d r^{2}-2 B d \tilde{\phi} d \tilde{t}+r^{2} d \tilde{\phi}^{2}
\end{aligned}
$$

As explained in 13], this metric and the Kerr metric differ in an important aspect, in that whereas the rotation for the Kerr black hole is bounded from above, here it is not, at least in principle. Thus, $B$ could be as large as desired.

The propagation of a sound wave in a barotropic inviscid fluid with irrotational flow, which is assumed to be the case, is described by the Klein-Gordon equation $\nabla_{\mu} \nabla^{\mu} \Phi=0$ for a massless field $\Psi$ in a Lorentzian acoustic geometry. Separating variables by the substitution

$$
\Phi(\tilde{t}, r, \tilde{\phi})=\sqrt{r} \Psi(r) e^{\mathrm{i}(m \tilde{\phi}-\omega \tilde{t})},
$$

implies that $\Psi(r)$ obeys the wave equation

$$
\Psi_{, \hat{r}_{*} \hat{r}_{*}}+Q \Psi=0
$$

where the generalized potential is given by

$$
Q \equiv\left(\hat{\omega}-\frac{\hat{B} m}{\hat{r}^{2}}\right)^{2}-\left(\frac{\hat{r}^{2}-1}{\hat{r}^{2}}\right)\left[\frac{1}{\hat{r}^{2}}\left(m^{2}-\frac{1}{4}\right)+\frac{5}{4 \hat{r}^{4}}\right] .
$$

The tortoise coordinate is defined by

$$
\frac{d \hat{r}_{*}}{d \hat{r}}=\Delta,
$$

where $\Delta \equiv\left(1-1 / \hat{r}^{2}\right)^{-1}$. Explicitly,

$$
\hat{r}_{*}=\hat{r}+\frac{1}{2} \log \left|\frac{\hat{r}-1}{\hat{r}+1}\right| .
$$

We have also performed the following rescaling: $\hat{r}=$ $r A / c, \hat{\omega}=\omega A / c^{2}, \hat{B}=B / A$. The rescaling effectively sets $A=c=1$ in the original wave equation, and picks units such that the acoustic horizon $\hat{r}_{H}=1$. From now on we shall omit hats in all quantities. The rescaled wave equation (7) will be the starting point of our analysis of QNMs. The characteristic QNMs of the rotating acoustic black hole can be defined in the usual way, imposing appropriate boundary conditions and solving the corresponding eigenvalue problem. Close to the sound horizon we seek solutions of equation (7) behaving as

$$
\Psi \sim e^{-\mathrm{i}(\omega-B m) r_{*}} .
$$

Classically, only ingoing waves - that is, waves falling into the acoustic black hole - should be present at the horizon. This means (according to our conventions on the time dependence of the perturbations) that we must choose the minus sign in the exponential. At spatial infinity the solutions of (7) behave as

$$
\Psi \sim e^{\mathrm{i} \omega r_{*}} .
$$

In this case we require that only outgoing waves (waves leaving the domain under study) should be present, and correspondingly choose the plus sign in the exponential. This boundary condition at infinity may be cause for objections. Indeed, no actual physical apparatus will be accurately described by these boundary conditions: a real acoustic black hole experiment will certainly not extend out to infinity. However, we may imagine using some absorbing device to simulate the "purely outgoing" wave conditions at infinity (for another example in which an absorbing device modeling spatial infinity could be required, cf. Section XI of [15] - in particular their Fig. $5)$.

The boundary conditions (11) and (12) are satisfied by only a discrete number of frequencies $\omega$, the QN frequencies $\omega_{Q N}$. The QN frequencies are in general complex numbers, and the imaginary part is usually negative, which means that perturbations die exponentially 
as time goes on (recall that the time dependence of the field is $e^{-i \omega t}$, and a negative imaginary part for $\omega$ means exponential decay with time). If all the QN frequencies have a negative imaginary part, we say that the system is stable. Note also that, for a given $m$ there will in general be an infinite number of $\omega_{Q N}$ satisfying the boundary conditions. We shall order these $\omega_{Q N}$ by imaginary part: the $\omega_{Q N}$ having smallest (in magnitude) imaginary part will be called the fundamental $(n=0)$ frequency, the one having the second lowest imaginary part will be the first overtone $n=1$, and so on.

\section{NUMERICAL PROCEDURE}

\section{A. Frobenius expansion}

In order to numerically obtain the QN frequencies, in the present investigation, we make use of Leaver's method [16, 17], which is known to yield excellent results.

Let us introduce a new independent radial variable $x$, defined as $x=r^{-1}$. In terms of the new variable $x$, the tortoise coordinate is then reduced to

$$
r_{*}=x^{-1}+\frac{1}{2} \ln (1-x)-\frac{1}{2} \ln (1+x) .
$$

The perturbation function $\Psi$ may be expanded around the horizon as

$$
\Psi=e^{i \omega x^{-1}}\left(\frac{1-x}{1+x}\right)^{-i(\omega-B m) / 2} \sum_{k=0}^{\infty} a_{k}(1-x)^{k},
$$

where $a_{0}$ is taken to be $a_{0}=1$. The expansion coefficients $a_{k}$ in equation (14) are determined via the fourterm recurrence relation (it's just a matter of substituting expression (14) in the wave equation (7)), given by

$$
\begin{aligned}
& \alpha_{0} a_{1}+\beta_{0} a_{0}=0, \\
& \alpha_{1} a_{2}+\beta_{1} a_{1}+\gamma_{1} a_{0}=0, \\
& \alpha_{k} a_{k+1}+\beta_{k} a_{k}+\gamma_{k} a_{k-1}+\delta_{k} a_{k-2}=0, k=2,3, \cdots,
\end{aligned}
$$

where

$$
\begin{aligned}
\alpha_{k}= & -8(1+k)(1+k+i B m-i \omega), \\
\beta_{k}= & 4\left\{1+5 k^{2}+m^{2}+k(5+4 i B m-8 i \omega)\right. \\
& \left.-4 i \omega-4 \omega^{2}+2 B m(i+2 \omega)\right\}, \\
\gamma_{k}= & 2\left(3-8 k^{2}-4 i k(B m-2 \omega)\right), \\
\delta_{k}= & -3-4 k+4 k^{2} .
\end{aligned}
$$

Since the asymptotic form of the perturbations as $r_{*} \rightarrow$ $\infty\left(r_{*} \rightarrow-\infty\right)$ is written in terms of the variable $x$ as

$$
e^{i \omega r_{*}} \sim e^{i \omega x^{-1}}\left(e^{-i(\omega-B m) r_{*}} \sim(1-x)^{-i(\omega-B m) / 2}\right),
$$

the expanded perturbation function $\Psi$ defined by equation (14) automatically satisfy the QNM boundary conditions if the power series converges for $0 \leq x \leq 1$. Making use of a Gaussian elimination [16], we can reduce the four-term recurrence relation to the three-term one, given by

$$
\begin{aligned}
& \alpha_{0}^{\prime} a_{1}+\beta_{0}^{\prime} a_{0}=0, \\
& \alpha_{k}^{\prime} a_{k+1}+\beta_{k}^{\prime} a_{k}+\gamma_{k}^{\prime} a_{k-1}=0, k=1,2, \cdots,
\end{aligned}
$$

where $\alpha_{k}^{\prime}, \beta_{k}^{\prime}$, and $\gamma_{k}^{\prime}$ are given in terms of $\alpha_{k}, \beta_{k}, \gamma_{k}$ and $\delta_{k}$ by

$$
\alpha_{k}^{\prime}=\alpha_{k}, \quad \beta_{k}^{\prime}=\beta_{k}, \quad \gamma_{k}^{\prime}=\gamma_{k}, \quad \text { for } k=0,1,
$$

and

$$
\begin{aligned}
\alpha_{k}^{\prime} & =\alpha_{k}, \\
\beta_{k}^{\prime} & =\beta_{k}-\alpha_{k-1}^{\prime} \delta_{k} / \gamma_{k-1}^{\prime}, \quad \text { for } k \geq 2 . \\
\gamma_{k}^{\prime} & =\gamma_{k}-\beta_{k-1}^{\prime} \delta_{k} / \gamma_{k-1}^{\prime}, \quad
\end{aligned}
$$

Now that we have the three-term recurrence relation for determining the expansion coefficients $a_{k}$, the convergence condition for the expansion (14), namely the QNM conditions, can be written in terms of the continued fraction as [17, 18]

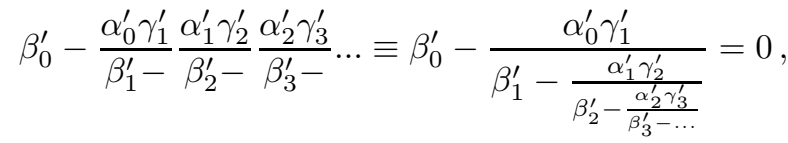

where the first equality is a notational definition commonly used in the literature for infinite continued fractions. Here we shall adopt such a convention.

An analysis of the large $k$ behavior of the expansion coefficients $a_{k}$ shows

$$
\lim _{k \rightarrow \infty} \frac{a_{k+1}}{a_{k}}=1 \pm \frac{(-2 i \omega)^{\frac{1}{2}}}{k^{\frac{1}{2}}}-\frac{3+4 i \omega}{4 k}+\cdots
$$

The series expansion (14) will converge uniformly only if the sign of the second term in the right hand side of (21) is chosen such that $\operatorname{Re}\left( \pm(-2 i \omega)^{\frac{1}{2}}\right)<0$, which will happen only for the $\mathrm{QN}$ frequencies $\omega$. In case that the convergency of the continued fraction (20) is not very good, one can use Nollert's technique to avoid this difficulty of the convergence [19]. In Nollert's technique, equation (21) plays an essential role.

\section{NUMERICAL RESULTS}

Our numerical results, which are all consistent with the results in [13], are shown in Figs. 18

(i) $m>0$ : In Fig. 14 we show results pertaining to perturbations having positive $m$, i.e., co-rotating waves. In Figs. 1:2 we show the real part of the QN frequencies for $m=1$ and $m=2$ modes respectively, as a function of the black hole rotation. Higher $m$ modes follow a similar pattern. One can see from these plots that for 


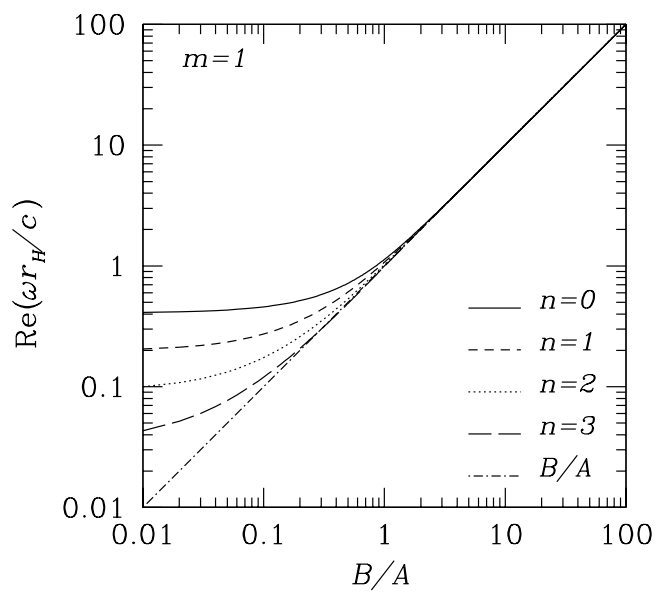

FIG. 1: The real part of the QN frequency as a function of the rotation parameter $B / A$, for several overtones of a $m=1$ mode. Here, $r_{H}=A / c$ is the horizon radius. Note how all the several lowest overtones "coalesce" in the high rotation regime, all growing linearly with $B / A$.

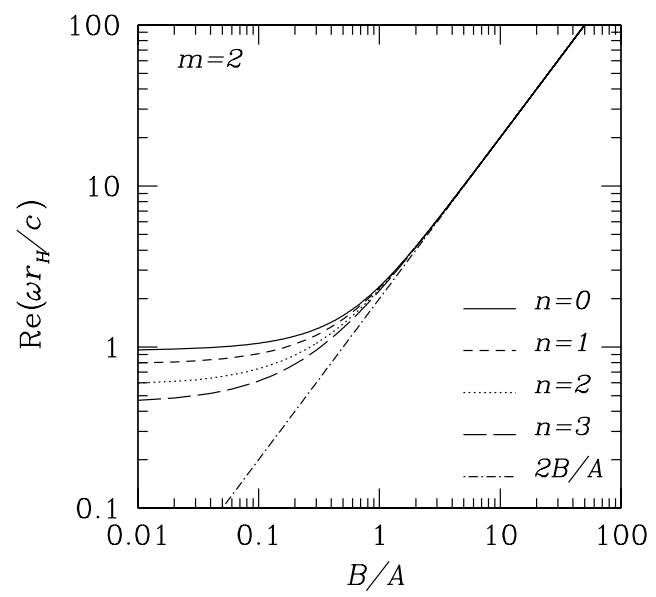

FIG. 2: The real part of the QN frequency as a function of the rotation parameter $B / A$, for several overtones of a $m=2$ mode. Again, all different overtones have the same behavior for high rotation.

low black hole rotation parameter $B$ the different overtones are clearly distinguished, but that as the rotation increases they tend to cluster and behave very similarly. For very large rotation $B$, all the overtones behave in the same manner, and in this high rotation regime the real part of the QN frequency scales linearly with the rotation. Indeed we find that the slope is also proportional to $m$ so that

$$
\operatorname{Re}\left[\omega_{Q N}\right] \simeq \frac{m B c^{2}}{A^{2}} \quad \text { as } B \rightarrow \infty, \text { for } m>0
$$

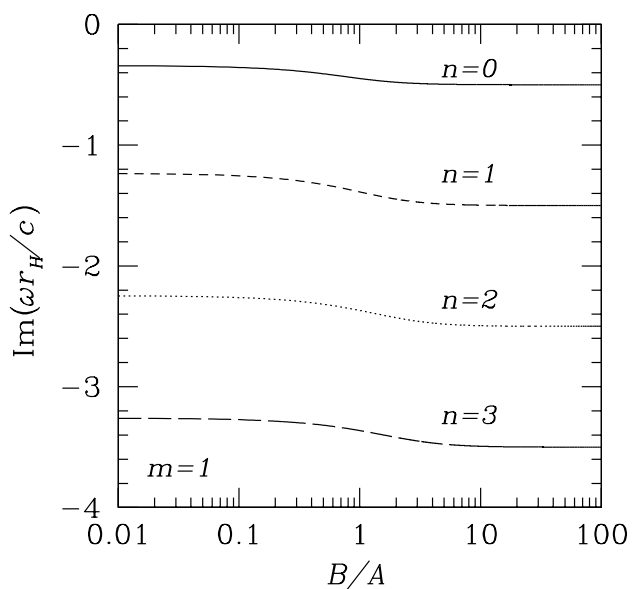

FIG. 3: The imaginary part of the QN frequency as a function of the rotation parameter $B / A$, for several overtones of a $m=$ 1 mode. It is clear from this plot that the imaginary part of the QN frequencies of $m>0$ modes is very insensitive to the rotation of the black hole.

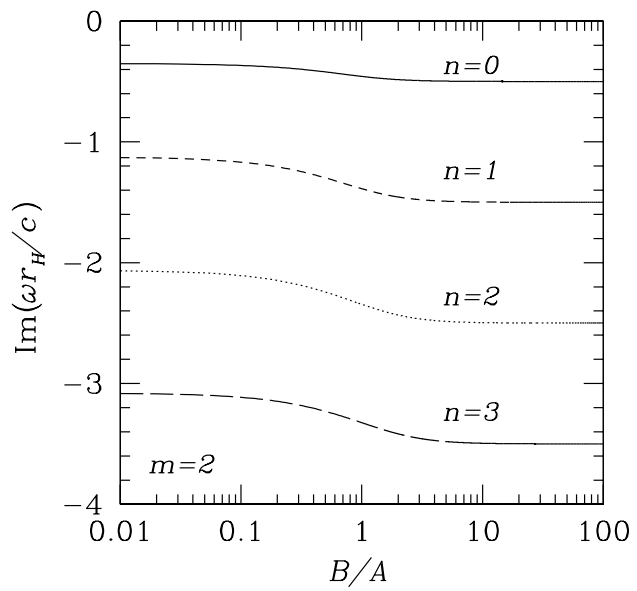

FIG. 4: The imaginary part of the QN frequency as a function of the rotation parameter $B / A$, for several overtones of a $m=$ 2 mode.

We notice that this behavior was already present in the WKB investigation in 13. In Figs. 34 we show the imaginary part of the QN frequencies as a function of the rotation parameter, for $m=1$ and $m=2$ respectively. Different overtones have different imaginary parts. Note also that for high $B$ the real part of the modes coalesce whereas the imaginary part does not. The magnitude of $\operatorname{Im}\left[\omega_{Q N}\right]$ increases with $B$, which was observed also in the WKB approach 13]. Thus, as the rotation increases the perturbation dies off quicker. This also means that the black hole is stable against $m>0$ perturbations, because 


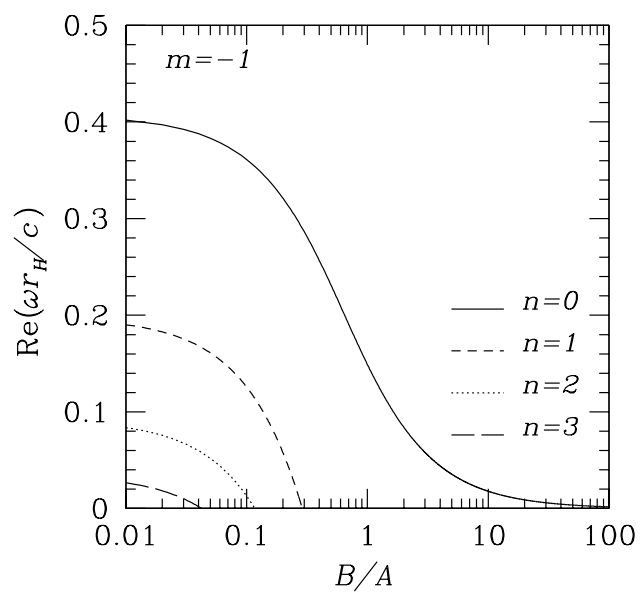

FIG. 5: The real part of the QN frequency as a function of the rotation parameter $B / A$, for several overtones of a $m=-1$ mode. Notice that for each overtone number $n$ there is a critical rotation at which the mode crosses the axis, i.e., there is a critical rotation $B / A$ at which the real part of the $\mathrm{QN}$ frequency is zero. Higher overtones cross the axis at a slower rotation. We have not been able to follow the mode beyond this point.

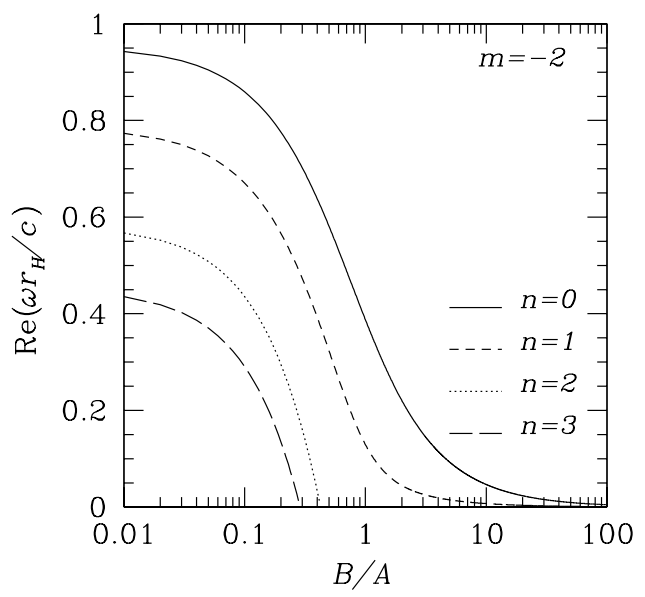

FIG. 6: The real part of the QN frequency as a function of the rotation parameter $B / A$, for several overtones of a $m=-2$ mode.

the imaginary part is always negative.

(ii) $m<0$ : In Figs. 58 we show results concerning perturbations having negative $m$, i.e., counter-rotating waves. The behavior of the QN frequencies for $m<0$ is drastically different from the $m>0$ perturbations. In Figs. [56] we plot the dependence of $\operatorname{Re}\left[\omega_{Q N}\right]$ as a function of the rotation of the black hole $B$. As $B$ increases the magnitude of the real part of the QN frequency de-

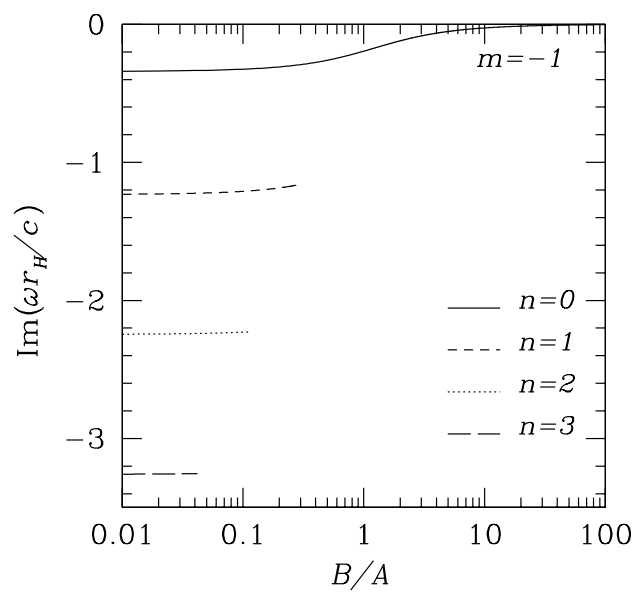

FIG. 7: The imaginary part of the QN frequency as a function of the rotation parameter $B / A$, for several overtones of a $m=-1$ mode. We have not been able to follow the modes beyond a certain critical point (defined as the rotation $B / A$ for which the real part of the $\mathrm{QN}$ frequency is zero- see figs. 5.6). Nevertheless, an judging by the modes we did manage to follow, namely the fundamental mode, it seems that $\operatorname{Im}\left[\omega_{Q N}\right]$ never crosses the axis, i.e., it is always negative, and therefore the mode is stable.

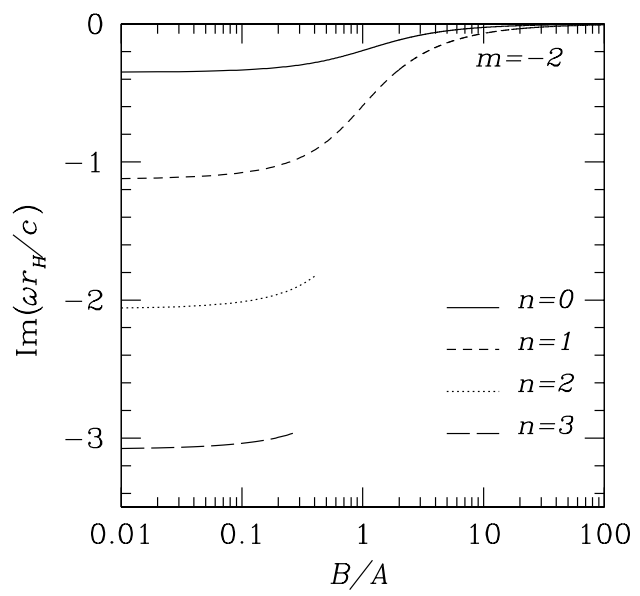

FIG. 8: The imaginary part of the QN frequency as a function of the rotation parameter $B / A$, for several overtones of a $m=$ -2 mode.

creases. The oscillation frequencies for the fundamental modes, labeled by $n=0$, indeed get close to the horizontal axis as $B$ goes to infinity. However, we haven't been able to track some overtone modes with negative $m$ for very high rotation since, as can be seen in Figs. [56] the real part of these modes eventually change sign. It is extremely difficult, using the method employed here, to compute modes having $\operatorname{Re}\left[\omega_{Q N}\right] \sim 0$. Nevertheless, 
supposing that (as the numerical studies for the fundamental modes indicate) the QN frequencies asymptote to zero for very large B, a WKB [20] analysis shows that $\omega_{Q N} \sim k / B$, where $k$ is some $m$-dependent constant. The imaginary part of the $\mathrm{QN}$ frequencies behaves in a similar manner, as seen in Figs. 78

(iii) $m=0$ : For circularly symmetric $(m=0)$ modes, our numerical method shows no sign of convergence. For $m=0$, the equation (7) can be written in the simpler form

$$
\Psi_{, \hat{r}_{*} \hat{r}_{*}}+\left(\omega^{2}-V\right) \Psi=0
$$

where

$$
V=\left(\frac{\hat{r}^{2}-1}{\hat{r}^{2}}\right)\left[-\frac{1}{4 \hat{r}^{2}}+\frac{5}{4 \hat{r}^{4}}\right] .
$$

The potential $V$ is not positive definite, and this precludes also a simple stability proof.

\section{CONCLUSIONS}

In this paper we have studied numerically the quasinormal modes of the $(2+1)$-dimensional draining bathtub metric, which describes a rotating acoustic black hole. Our results indicate that this is a metric stable against small perturbations, although one would like to have also an analytical proof of this statement. Notice that this proof, would most certainly encompass also the the stability of the usual general relativistic black holes against perturbations of a charged scalar field; In fact, for such a field it is possible to show 21] that the generalized potential goes like $(\omega-e q / r)^{2}-V$, where $e$ is the scalar field charge, $q$ the charge of the black hole, and $V$ an $\omega$-independent potential. This is of the same form as the generalized potential dealt with here, equations (23)-(8). Ever since the first studies on the Kerr geometry (see for example [22], and 23] for numerical results regarding the QN frequencies of rotating Kerr black holes), one knows that it is extremely difficult to prove stability when $\omega^{2}-Q$ is not positive definite (or when it is $\omega$-dependent), and therefore the special case $Q=(\omega-e q / r)^{2}-V$ could shed some light on this problem. To conclude, we would like to draw attention to the fact that the results presented here are similar in many respects to the results concerning higher dimensional rotating black holes [24].

\section{Acknowledgements}

This work was partially funded by Fundação para a Ciência e Tecnologia (FCT) - Portugal through project CERN/FNU/43797/2001. V.C. acknowledges financial support from FCT through grant SFRH/BPD/2003. S.Y. is supported by the Grant-in-Aid for the 21st Century COE "Holistic Research and Education Center for Physics of Self-organization Systems" from the ministry of Education, Science, Sports, Technology, and Culture of Japan.
[1] W. G. Unruh, Phys. Rev. Lett. 46, 1351 (1981).

[2] T. Jacobson, Phys. Rev. D 48, 728 (1993).

[3] W. G. Unruh, Phys. Rev. D 51, 2827 (1995).

[4] M. Visser, Class. Quantum Grav. 15, 1767 (1998).

[5] U. R. Fischer, G. E. Volovik, Int. J. Mod. Phys. D 10, 57 (2001).

[6] C. Barceló, S. Liberati, S. Sonego and M. Visser, gr-qc/0408022

[7] B. Reznik, Phys. Rev. D 62, 0440441 (2000).

[8] L. J. Garay, J. R. Anglin, J. I. Cirac and P. Zoller, Phys. Rev. A 63, 023611 (2001).

[9] C. Barceló, S. Liberati and M. Visser, Class. Quantum Grav. 18, 1137 (2001).

[10] V. Cardoso, " Quasinormal Modes and Gravitational Radiation in Black Hole Spacetimes", PhD thesis, Instituto Superior Tecnico, Universidade Técnica de Lisboa, December 2003, gr-qc/0404093

[11] M. Visser and S. E. Ch. Weinfurtner; gr-qc/0409014

[12] S. Basak and P. Majumdar, Class. Quant. Grav. 20, 3907 (2003).

[13] E. Berti, V. Cardoso and J. P. S. Lemos, Phys. Rev. D, in press; gr-qc/0408099

[14] S. Lepe and J. Saavedra, gr-qc/0410074
[15] R. Schützhold, W. G. Unruh, Phys. Rev. D 66, 044019 (2002).

[16] E. W. Leaver, Phys. Rev. D 41, 2986 (1990).

[17] E. W. Leaver, Proc. R. Soc. London A402, 285 (1985).

[18] W. Gautschi, SIAM Rev. 9, 24 (1967).

[19] H.-P. Nollert, Phys. Rev. D 47, 5253 (1993).

[20] B. F. Schutz and C. M. Will, Astrophys. Journal 291, L33 (1985); C. M. Will and S. Iyer, Phys. Rev. D 35, 3621 (1987); R. A. Konoplya, Phys. Rev. D 68, 024018 (2003).

[21] S. Hod and T. Piran, Phys. Rev. D 58, 024017 (1998).

[22] V. P. Frolov and I. D. Novikov, Black-hole physics (Kluwer, Dordrecht, 1998).

[23] E. W. Leaver, Proc. R. Soc. London A402, 285 (1985). H. Onozawa, Phys. Rev. D 55, 3593 (2003); E. Berti and K. D. Kokkotas, Phys. Rev. D 68, 044027 (2003); E. Berti, V. Cardoso, K. D. Kokkotas and H. Onozawa, Phys. Rev. D 68, 124018 (2003); E. Berti, V. Cardoso and S. Yoshida, Phys. Rev. D 69, 124018 (2004).

[24] E. Berti, K. D. Kokkotas and E. Papantonopoulos, Phys. Rev. D 68, 064020 (2003); S. Yoshida, V. Cardoso, G. Siopsis and S. Musiri, in preparation. 